\title{
Quantifying age-related differences in human reaching while interacting with a rehabilitation robotic device
}

\author{
Vivek Yadav ${ }^{\mathrm{a}}$, James P. Schmiedeler ${ }^{\mathrm{b} *}$, Sharon McDowell ${ }^{\mathrm{c}}$ and Lise Worthen-Chaudhari ${ }^{\mathrm{c}}$ \\ ${ }^{a}$ Department of Mechanical Engineering, The Ohio State University, Columbus, Ohio, USA; ${ }^{b}$ Department of Aerospace and Mechanical \\ Engineering, University of Notre Dame, Notre Dame, Indiana, USA; ${ }^{c}$ Department of Physical Medicine \& Rehabilitation, The Ohio State \\ University, Columbus, Ohio, USA
}

(Received 29 January 2010; final version received 24 November 2010)

\begin{abstract}
New movement assessment and data analysis methods are developed to quantify human arm motion patterns during physical interaction with robotic devices for rehabilitation. These methods provide metrics for future use in diagnosis, assessment and rehabilitation of subjects with affected arm movements. Specifically, the current study uses existing pattern recognition methods to evaluate the effect of age on performance of a specific motion, reaching to a target by moving the end-effector of a robot (an X-Y table). Differences in the arm motion patterns of younger and older subjects are evaluated using two measures: the principal component analysis similarity factor $\left(S_{P C A}\right)$ to compare path shape and the number of Fourier modes representing $98 \%$ of the path 'energy' to compare the smoothness of movement, a particularly important variable for assessment of pathologic movement. Both measures are less sensitive to noise than others previously reported in the literature and preserve information that is often lost through other analysis techniques. Data from the $S_{P C A}$ analysis indicate that age is a significant factor affecting the shapes of target reaching paths, followed by reaching movement type (crossing body midline/not crossing) and reaching side (left/right); hand dominance and trial repetition are not significant factors. Data from the Fourier-based analysis likewise indicate that age is a significant factor affecting smoothness of movement, and movements become smoother with increasing trial number in both younger and older subjects, although more rapidly so in younger subjects. These results using the proposed data analysis methods confirm current practice that age-matched subjects should be used for comparison to quantify recovery of arm movement during rehabilitation. The results also highlight the advantages that these methods offer relative to other reported measures.
\end{abstract}

Keywords: reaching; principal component analysis; smoothness; rehabilitation

\section{Introduction}

Evaluating human arm motion is important for diagnosis, assessment and rehabilitation of patients suffering from arm movement disorders, such as those that often result from stroke. Most current clinical motor-deficit evaluations measure impairment and function qualitatively, employing ordinal scales, binary decisions (i.e. patient can/cannot perform the task) or qualitative descriptions. Examples include the FIM ${ }^{\mathrm{TM}}$ (Keith et al. 1987) and the Wolf Motor Function Test (Morris et al. 2001). Although these functional tests examine patient independence, they do not quantify function or capture the shape, variability or smoothness of movement paths used to complete a task key elements for optimising evaluation and rehabilitation planning (Colombo et al. 2005). Thus, there is a need for quantifiable assessment techniques that are both easy to use in the clinic and able to capture hand-path geometry, performance variability and motion smoothness.

Rehabilitation robots provide one means of collecting the kinematic data required for such assessment, with sub- jects holding the handle of a device while executing arm motions. A variety of currently available devices are being used for this purpose, and the effectiveness of such devices in rehabilitation of lost motor function is well documented in the literature (Burgar et al. 2000; Volpe et al. 2000; Lum et al. 2002). An open question is how best to analyse the data collected with these robots in order to quantify hand-path geometry and motion smoothness. It is somewhat standard to make comparisons with data collected from subjects who have no arm disorders under similar experimental conditions. This approach both requires selection of appropriate data analysis methods and introduces the question of whether these subjects must be age-matched to those with arm disorders for comparison. In terms of data analysis methods, the present study suggests a novel application of Principal Component Analysis (PCA), the similarity index (Yang and Shahabi 2004), to compare hand-path shape between groups and a Fourier-based measure to quantify smoothness of motion. The effectiveness of these methods is evaluated by comparing differences in arm motions

*Corresponding author. Email: schmiedeler.4@nd.edu 
of younger and older subjects while interacting with an unpowered robotic device, an $\mathrm{X}-\mathrm{Y}$ table, and comparing results to those previously reported in the literature.

Common approaches to quantifying hand-path shape include normalising path length by the length of a straightline path and measuring the mean absolute value of the distance between the actual path and a straight line. These may be appropriate for experiments in which subjects are instructed to follow straight-line paths (as in Colombo et al. 2005 and Colombo et al. 2007); however, they are not applicable to free reaching exercises and more functional activities in which hand paths are not necessarily straight. Additionally, these metrics quantify only deviations from a straight line in an absolute sense, so they do not directly indicate path shape. For example, two hand paths of vastly different shape might exhibit identical values for either metric, so they would be indistinguishable with this method of data analysis. PCA is an alternative analysis technique that can give a more direct measure of hand-path shape. PCA has been used to analyse joint angle co-ordination in upper extremity reaching (Forner-Cordero et al. 2005), Electromyographic (EMG) and kinematic data in functional upper extremity tasks (Lum et al. 2009) and ground reaction force patterns in walking (Muniz and Nadal 2007). In these and most similar studies, though, PCA is applied specifically to reduce the dimensionality of the data set and obtain a compact representation of human motion. In contrast, the present work employs PCA to extract the important modes that describe hand-path shape and then to quantify similarity between these modes for subjects in different groups by using an inner product.

The dominant approach to quantifying motion smoothness is measuring jerk, the third time derivative of position, with lower jerk values corresponding to smoother motions (Flash and Hogan 1985). Differentiating position data three times, though, amplifies any noise in the original signal, and using a low pass filter to eliminate the effects of the noise can cause loss of data. One less differentiation is required with a metric that counts the number of times the acceleration profile changes sign (Teulings et al. 1997), but it is still subject to issues with noise. Alternatives requiring at most one differentiation include a count of the number of peaks in speed (Brooks et al. 1973), the mean speed normalised by the peak speed (Rohrer et al. 2002), the Movement Arrest Period Ratio (Beppu et al. 1984) and a 'tent' metric that measures the ratio of the area under the speed curve to the area under a single acceleration/deceleration phase curve scaled to share the same peak speed (Rohrer et al. 2002). One limitation of each of these alternatives, similar to that identified for the path shape metrics above, is that vastly different velocity profiles may be indistinguishable if they yield identical values for the smoothness metric. For example, a velocity profile with a single large peak and three smaller peaks is considered equally smooth with any velocity profile having four extremely large peaks according to the metric that simply counts velocity peaks. Similar examples apply to the other three alternatives. Celik et al. (2010) use a method of measuring motion smoothness that is relatively free from these errors by computing the correlation coefficient between the velocity profile traced by a subject and the minimum jerk trajectory computed from the movement time and amplitude information (Flash and Hogan 1985). This method, however, assumes that the ideal path between the initial and final position is a straight line and is traced with a minimum jerk profile. In contrast, spectral smoothness measures have been used in data reconstruction applications to manage the compromise between fidelity to the original data and smoothness in the approximation (Kitagawa and Gersch 1985). The present work applies these techniques that consider the entire shape of the curve to quantify smoothness of the velocity profile in reaching. The general approach is to approximate the time-series data as a linear combination of some basis functions and to then compute the smoothness based on the coefficients of the approximating series, a Fourier series in this case.

A number of studies involving directed reaching, drawing and other fine motor performance have shown that elderly populations, compared with younger populations, exhibit differences in their arm movements that include reduced speed (Smith et al. 1999; Ketcham et al. 2003; Nicoletti et al. 2005; Lee et al. 2007), reduced smoothness (Ketcham et al. 2002), and reduced ability to adapt to visuomotor rotations (Buch et al. 2003; Heuer and Hegele 2008). According to Ketcham et al. (2002), wherein pointing motions to targets requiring different movement amplitudes were performed on a tablet, a typical aiming motion consists of a primary movement that takes the pointer to the vicinity of the target and a secondary movement that takes the pointer to the final location. When the movement amplitude was increased, elderly subjects did not increase the distance traveled in the primary submovement and did not scale the velocity to the same magnitude as younger subjects. Both factors caused older subjects to spend a larger fraction of the total movement time in the secondary motion, resulting in both longer movement times and less smooth velocity profiles. Notably, though, the shapes of the paths traced by the elderly and younger groups were not significantly different. Similarly, Lee et al. (2007) identified no age-related deteriorations in the accuracy of line tracing when subjects were asked to trace a line at different cycling frequencies. The older subjects in this study, however, did perform the motions more slowly than the younger subjects. Comparison of individual muscle torques and their contributions to the net torque at each joint revealed that the older subjects changed their joint co-ordination strategies to reduce the muscle torque requirements, resulting in less smooth movements.

The ability to adapt to novel visuo-motor transformations can be considered an indicator of the well-being of the motor control system and is subject to age-related 
decline. For experiments in which subjects moved a digitising pen toward visually presented targets and visual feedback was provided as a rotated screen cursor, elderly subjects (average age of 73.3 years) exhibited reduced adaptation compared with younger subjects for sudden large rotations, but no age-related differences were found when the large visuo-motor rotation was introduced incrementally (Buch et al. 2003). In a similar study, no age-related differences in visuo-motor adaptations were observed in older subjects who were below the retirement age (average age $=$ 56.8 years) (Heuer and Hegele 2008). Taken together, these results indicate that there may be a threshold for adaptation that depends on the age of the subjects.

Not surprisingly, the same three age-related effects (reduced speed, reduced smoothness, and reduced motor adaptation ability) have been observed in subjects performing arm motions while interacting with a robotic device. Cesqui et al. (2008) report reduced speed and smoothness for older subjects (average age $=72$ years) executing arm motions while holding the handle of a manipulandum applying novel velocity-dependent force fields. Also, these older subjects required more trials to adapt to the force fields as they employed a strategy that emphasised reducing the required joint torques over achieving the same arm kinematics as in the unperturbed case.

The data analysis methods developed in the present study overcome some of the issues with the existing alternatives. This study is the first step in developing and validating these methods to quantify human arm movements by using a robotic system, specifically for diagnosis and assessment during the acute phase of stroke recovery. To be able to quantify the changes in motion patterns of stroke patients over the period of rehabilitation, it is important to understand 'normal' motion patterns used with a particular robotic device. This study is designed to assess the novel data analysis techniques in establishment of these 'normal' motion patterns for subjects manipulating the handle of an unpowered $\mathrm{X}-\mathrm{Y}$ table by answering the following questions: Are hand-path shape and motion smoothness, measured via metrics developed in this study, different for younger and older subjects? If so, in what ways are they different? Furthermore, since subjects are not initially accustomed to performing reaching motions while holding the handle of the device, they must adapt their motion strategies to the task. By studying the adaptation of the motion patterns, the adaptation abilities of the two groups can be compared. The older group, composed of those over 50 years, has been chosen such that the ages are similar to those of typical stroke patients.

\section{Materials and methods}

\subsection{Subjects}

A total of 16 right-handed subjects volunteered for the experiments: 8 ( 6 men, 2 women) from a younger population

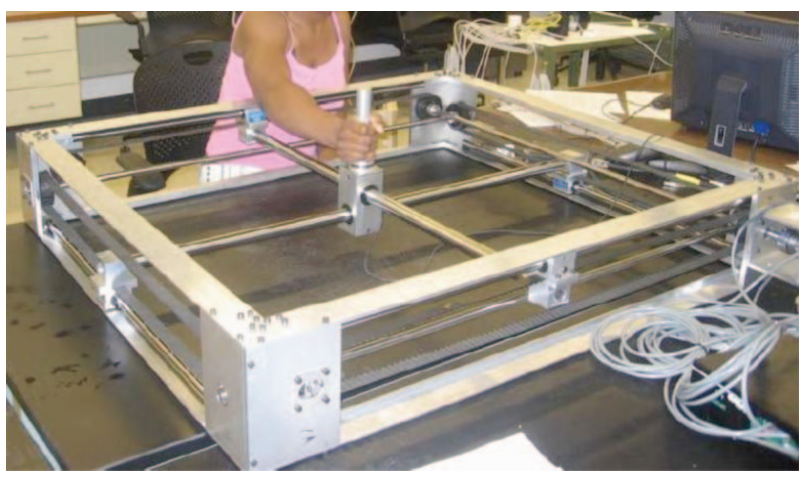

Figure 1. The experimental apparatus, the unpowered X-Y table.

(ages 20-30) of mean age 24.25 years and standard deviation 3.06 years, and 8 (4 men, 4 women) from an older population (ages 52-60) of mean age 55.63 years and standard deviation 2.97 years. Subjects with injuries affecting their arm motion or prior neurological injuries were excluded from the study. The experiments were conducted according to the guidelines of the Institution Review Board of The Ohio State University.

\subsection{Apparatus}

The experiments were performed using an unpowered robotic device, the $\mathrm{X}-\mathrm{Y}$ table (Figure 1). The $\mathrm{X}-\mathrm{Y}$ table is a planar prismatic - prismatic manipulator that has two perpendicular cross shafts connected by a crossbar bearing housing. These perpendicular cross shafts are connected to two encoders via timing belts and pulleys. The encoders measure the rotation of the pulleys, which can then be used to calculate the location of the cross shafts relative to a fixed point on the table. The encoders have an accuracy of 1,000 counts per revolution. As the pitch diameter of the pulleys is 2.387 inches $(6.063 \mathrm{~cm})$, the precision for measurement of position is $\frac{\pi d}{1000} \approx 7.5 \times 10^{-3}$ inches $\left(19 \times 10^{-3} \mathrm{~cm}\right)$. Additional details pertaining to this custom $\mathrm{X}-\mathrm{Y}$ table are provided by Crear (2006).

To avoid subject fatigue, this $\mathrm{X}-\mathrm{Y}$ table was mounted on pillars upside down relative to Figure 1 atop a highlow table so that the surface of the high-low table could support the weight of the subject's arm. The height of this table was adjusted for subjects of different heights. To avoid discomfort and decrease friction due to sliding on the table surface, each subject rested his/her arm in a splint attached to the crossbar bearing housing of the X-Y table through a handle. The handle was designed for rapid connection/disconnection of the splints for the left and right arms. The splint with the handle attachment is shown in Figure 2. To avoid injury due to pinching, the moving parts were covered by a plexiglass sheet. The full experimental apparatus is shown in Figure 3.

The subject completed the tasks of the experiment by watching how his/her movements of the $\mathrm{X}-\mathrm{Y}$ table handle 


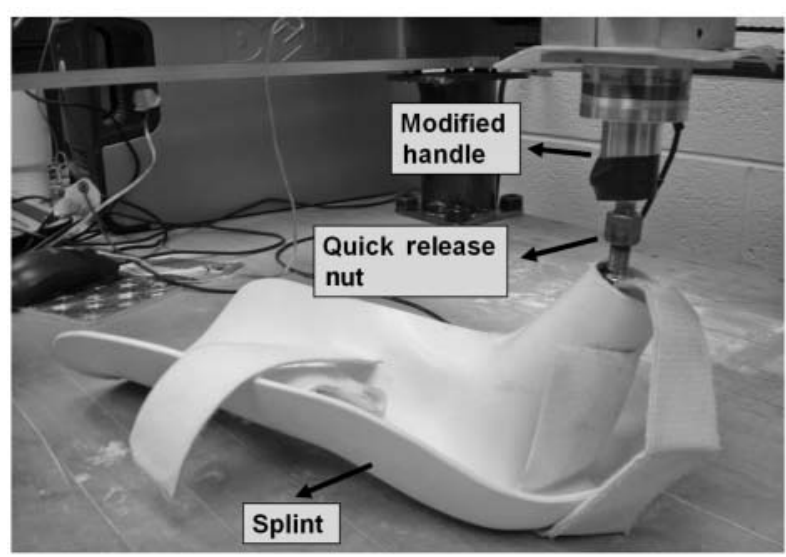

Figure 2. Complete assembled handle and splint.

caused a circle to move relative to other objects presented on an elevated computer screen at the rear of the X-Y table. The workspace boundary of the $\mathrm{X}-\mathrm{Y}$ table was displayed as a fixed rectangle on the screen. Within this rectangle, a fixed green circle (diameter $\approx 1 / 20$ th the length of the rectangle) was displayed at what is considered the 'home' position of the handle within the experiment. A smaller blue circle (diameter $\approx 1 / 40$ th the length of the rectangle) was displayed to indicate the position of the handle within the X-Y table workspace. Finally, the target locations were presented as red circles (same diameter as the green circle) that appear throughout the course of the experiment. When the blue circle representing the handle moved over the green home position circle or the red target circle, the bottom colour was obscured. This enabled the subject to confirm that he/she had reached the desired destination and was why the blue circle is smaller in diameter.

\subsection{Experimental protocol}

The subject was first strapped into a wheelchair with cotton gait belts to eliminate trunk movement and to ensure that nearly all of the arm movement was due to shoulder

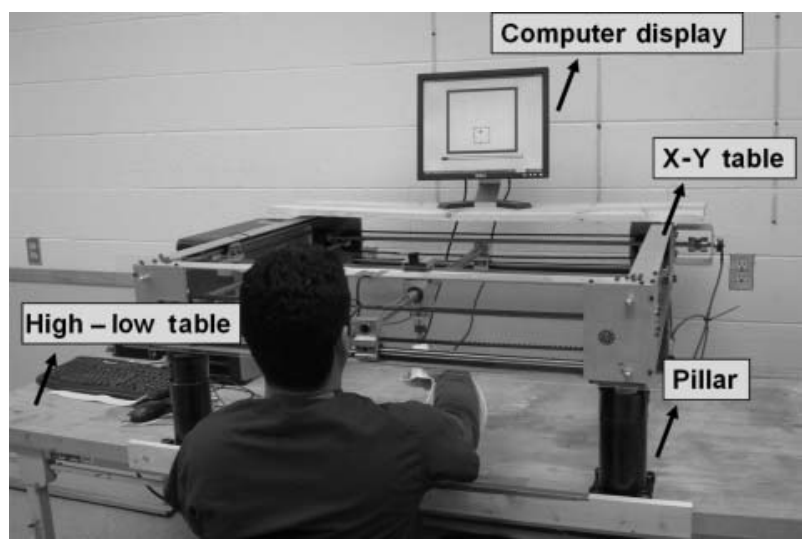

Figure 3. A volunteer using the experimental apparatus. and elbow joint motions. The wheelchair was positioned with the centre of the X-Y table directly in front of the subject. The height of the high-low table and the location of the wheelchair were adjusted so that the subject's arm was horizontal when in the splint resting on the X-Y table. The subject was asked to move his/her arm to ensure that the shoulder did not contact the X-Y table. Nine of the subjects performed the experiment with their left arms first, and the other seven performed the experiments with their right arms first. The arm with which the subject first performed the experiment is referred to as the 'first arm' and the other as the 'second arm' in the remainder of the paper.

The subject's first arm was strapped to the splint, and the experiment consisted of the following procedures:

- Initialisation: The subject was asked to take the handle to the 'home' position of the table to initialise the encoders. The home position was the centre-bottom location of the $\mathrm{X}-\mathrm{Y}$ table - the point on the table directly in front of the subject.

- Measurement: The subject's forearm length was measured from the bony landmark on the distal head of the ulna to the proximal head of olecranon with a measuring tape. (This measurement was used to determine the location of the targets for the reaching tasks.)

- Play: The subject was asked to hold the handle of the X$\mathrm{Y}$ table and move it around to familiarise himself/herself with the device and the visual display of his/her movements.

- Reaching: The subject was asked to extend his/her arm toward targets presented one at a time on the computer screen and then return to the home position. The targets were arranged symmetrically about the midline of the $\mathrm{X}-\mathrm{Y}$ table. The target locations were scaled according to the subject's forearm length $\left(l_{f a}\right)$ with co-ordinates of $\left( \pm 0.47 l_{f a}, 0.80 l_{f a}\right)$ relative to the origin at the home location. Thus, the plus and minus indicate right and left of the midline of the X-Y table. The presentation of the targets was randomised, and the subject made a total of five reaches toward each target. The subjects were simply asked to move the hand to the target location presented on the screen without instruction as to how to move the arm. The goal was to study 'natural' reaching movements of the subjects while holding the handle of the device, independent of whether those resulted in straight or curved hand paths. Therefore, no instructions regarding the shape of the path were given to the subjects.

Once all of the tests with the first arm were completed, the splint was changed on the X-Y table, and the second arm was strapped into the setup. The same set of experiments was repeated with the second arm. 


\subsection{Data analysis}

The X-Y handle position data computed from the encoder data were truncated on the basis of the maximum velocity of reaching. The point before which the velocity remained less than $2 \%$ of the maximum velocity was considered the start of motion. Completion was determined by the point at which the velocity fell and remained below $2 \%$ of the maximum velocity. As half of the motions were performed toward a target on the left and half toward the right, the motions toward the left were reflected about the Y-axis to eliminate the effect of performing motion toward targets in different directions. Since the target locations were based on the individual subject's arm length, the $\mathrm{X}-\mathrm{Y}$ data were normalised by the arm length. With the velocity-based truncation, in general each motion contained a different number of data points. Instead of using interpolation techniques to achieve an equal number of data points, a functional representation of the X-Y data for each motion was obtained by fitting a fifth order piecewise continuous spline with break points placed after every 10 data points. These polynomials were the lowest order polynomials that approximated the position data accurately.

The processed data were analysed according to three metrics. The first metric, $S_{P C A}$ (Yang and Shahabi 2004), quantified the similarity between two groups of reaching paths, where the division into groups was based upon the categories of (1) subject age, (2) direction of reaching, (3) use of the dominant/non-dominant arm, (4) reaching to the ipsilateral/contralateral hemifield, (5) reaching with the first/second arm and (6) trial number. This metric was used to identify the important factors among these six affecting the reaching motion patterns. Then, the second metric quantified the variability in reaching, computed as the signed area between the path traced by the subject and an average path computed for the corresponding combination of important factors obtained from the first metric. The third metric was a measure of the smoothness of the velocity profile.

\subsection{1. $S_{P C A}$}

The PCA similarity factor $S_{P C A}$ is defined between two matrices of the same number of columns but not necessarily the same number of rows (Krzanowski 1979). $S_{P C A}$ firstly obtains the principal components for each matrix and selects the first few principal components on the basis of some heuristic, such as the first $k$ principal components whose variances represent $99 \%$ of the total variance (Krzanowski 1979). $S_{P C A}$ then computes the similarity between the first $k$ principal components of the two matrices as an inner product.

To compare the paths of two groups 1 and 2, the path data for reaching motions of each group were arranged in a matrix whose rows represent different reaching motions and whose columns represent the path information ( $\mathrm{Y}$ at 100 equally spaced values of $\mathrm{X}$ ). Principal components for the two matrices were extracted. Suppose $n$ principal components from each of the groups represented more than $99 \%$ of the total variance. Similarity between the two sets of principal components was calculated by taking the inner product between the two sets.

$$
\begin{aligned}
S_{P C A}= & \frac{\operatorname{trace}\left(P C_{1} P C_{2}^{T} P C_{2} P C_{1}^{T}\right)}{n} \times 100 \\
= & \frac{\sum_{i=1}^{n} \sum_{j=1}^{n}<P C_{1, i}, P C_{2, j}>^{2}}{n} \times 100,
\end{aligned}
$$

where $P C_{1, i}$ and $P C_{2, i}$ represent the $i^{\text {th }}$ principal components of the 1 st and 2 nd groups. The principal components are arranged such that the corresponding eigenvalues are in descending order. The inner product $\langle a, b\rangle$ is defined as the dot product between the vectors $a$ and $b$, so it varies between 1 for identical principal components and 0 for orthogonal principal components. Therefore, the $S_{P C A}$ value varies between 0 and 100, with proximity to 100 indicating greater similarity between the two data sets. Krzanowski (1979) calculates the similarity as the inner product of the principal components alone, i.e. the numerator of the summation in Equation (1). As the number of components required to capture more than $99 \%$ of covariance can be different for different groups in the present work, the $S_{P C A}$ value was normalised to vary between 0 and 100 to aid in comparing performance of different groups.

$S_{P C A}$ was used to identify the important factors that affect the paths of the subjects. The reaching paths were grouped according to the following categories:

- Age group (younger/older): denotes the age group of the subjects.

- Direction (left/right): denotes the direction in which a motion is performed (to the left or right).

- Dominance (dominant/non-dominant): denotes the arm with which the motion is performed. As all subjects were right-handed the dominant arm is equivalent to the right arm.

- Type (contralateral/ipsilateral): denotes whether the movement was made to the contralateral or ipsilateral hemifield - a combination of the direction and dominance categories.

- Order (first arm/second arm): denotes whether the movement was made with the first or second arm used in the study.

- Trial number (1/2/3/4/5): denotes the trial (meaning repetition) number of the motion.

To determine if the $S_{P C A}$ value for the two groups, say $g_{1}$ and $g_{2}$, indicated relevant differences between them, the 
similarity within each group was computed and compared with the similarity between groups. The intragroup similarity was calculated by dividing a single group's data in half and computing the similarity between the two data sets. This process was repeated four times with different divisions of the data, and the average of the four $S_{P C A}$ values was taken as the estimate of the intragroup similarity.

\subsubsection{Variability in path}

With the important factors identified using the $S_{P C A}$ values, the reaching motions of the subjects were grouped into sets, each corresponding to a particular combination of the significant factors. Thus, $S_{P C A}$ enabled like motions to be binned together for calculation of a representative average motion, relative to which individual path variability could be quantified. The principal components representing $99 \%$ of the covariance were used to compute the average path for each set.

$$
Y_{g, a v g}=\sum_{j=1}^{m} \sum_{i=1}^{n} \frac{a_{i j}}{m} P C_{g, i}
$$

where $g$ corresponds to the set, $m$ is the number of reaching paths in $g, n$ is the number of principal components required to capture $99 \%$ of the covariance, $a_{i j}$ is the coefficient of the $i$ th principal component $P C_{g, i}$ for the $j$ th path and $Y_{g, a v g}$ is the average path corresponding to set $g$. The signed area $A_{j}$ between each individual path $j$ traced by a subject, and the average path of the corresponding set was computed to provide a measure of variability. Then, statistical analysis (detailed in Section 2.4.4) with $A_{j}$ as a response variable was used to identify the important factors affecting the variability in the reaching motions.

\subsubsection{Velocity profile smoothness}

The tangential velocity information was obtained by numerically differentiating the position data with respect to time once and then filtering with a moving average filter with a span of 5 . This velocity profile was normalised with respect to the duration of the motion, and a Fourier series with 500 terms was fit to the normalised velocity profile. Smoothness was quantified by computing $N^{*}$, the number of modes that capture most of the 'energy' in the waveform (98\% for this data), where the energy of a particular mode is defined as the square of its coefficient. Therefore, the energy in the first $N$ modes is

$$
\% \text { energy }=\frac{\sum_{i=1}^{N} a_{i}^{2}}{\sum_{i=1}^{500} a_{i}^{2}}
$$

where $a_{i}$ is the coefficient of the $i$ th mode. $N^{*}$ is calculated by increasing $N$ in Equation 3 until the percentage of energy exceeds $98 \%$. A lower value of $N^{*}$ indicates a smoother velocity profile. Statistical analysis with $N^{*}$ as a response variable was performed to identify the important factors that affect motion smoothness (Section 2.4.4).

\subsubsection{Statistical analysis}

Statistical analysis was carried out with $A_{j}$ (quantifying the variability in the reaching paths) and $N^{*}$ (quantifying the velocity profile smoothness) as response variables. Linear regression (MINITAB's GLM) was used to perform the statistical analysis. Age group, direction, dominance, movement type and order were included as factors for the statistical analysis. Since the subjects of the experiments came from a larger population, subjects were incorporated into the model as a random effect nested within age group. As more movements are performed, there is potential for learning effects associated with the subject adapting to the motion and to the X-Y table. This effect is included in the statistical analysis by including trial number as a covariate. In combination, these led to a nested-analysis of covariance (ANCOVA) model for determining the important factors affecting the metrics. ANCOVA has been used in other work to study between-subject and within-subject effects with practice incorporated into the model as a covariate and age group as a factor (Olivier et al. 1998). Bonferroni post hoc tests were carried out between important factors to perform pairwise comparisons.

\section{Results}

\section{1. $S_{P C A}$ application}

Table 1 lists the $S_{P C A}$ values for the intergroup comparisons. Since these values exceed 99 in the dominance and order categories, indicating strong intergroup similarity, no intragroup similarities were computed for these categories. The intragroup $S_{P C A}$ values for the age group, direction and type categories are listed in Table 2, and in each case, the corresponding intergroup similarity in Table 1 has a lower value. These results suggest that the important factors that affect the reaching motion patterns are the age group, direction and type. Neither the dominance of the arm with which

Table 1. Intergroup $S_{P C A}$ values comparing paths traced by subjects corresponding to the respective groups.

\begin{tabular}{lc}
\hline Category & $S_{P C A}$ \\
\hline Age group: older vs. younger & 90.3 \\
Direction: left vs. right & 96.6 \\
Dominance: dominant vs. non-dominant & 99.2 \\
Type: contralateral vs. ipsilateral & 96.2 \\
Order: first arm vs. second arm & 99.1 \\
\hline
\end{tabular}


Table 2. Intragroup $S_{P C A}$ values computed for individual groups.

\begin{tabular}{ll}
\hline Group & $S_{P C A}$ \\
\hline Older & 98.56 \\
Younger & 97.54 \\
Left & 98.37 \\
Right & 98.91 \\
Contralateral & 98.64 \\
Ipsilateral & 99.02 \\
\hline
\end{tabular}

the movements were performed, nor the order in which the arm was used had a significant effect on the paths traced by the subjects. Figures 4 and 5 contain plots showing all of the paths (grey) and their averages (black) for four of the groups listed in Table 1. Note that the averages are provided strictly for visualisation and are not involved in the $S_{P C A}$ analysis. The Y-data in Figure 4 is normalised by the arm length, while the X-data, likewise normalised by the arm length for the analysis, is scaled in the plot to vary between 0.5 and 1 for compactness of presentation. The paths for the older and younger subjects in Figure 4(a) indicate that the older subjects in general traced more curved paths than the younger subjects. Similarly, Figure 4(b) shows that paths were more curved when subjects reached to the right side than when they reached to the left side, as were paths for reaching in the ipsilateral hemifield compared with the contralateral hemifield, as shown in Figure 5(a). In contrast, paths for the first and second arm, shown in Figure 5(b), were in general quite similar, as were paths for dominant and non-dominant arms, for which no plots are included here.

\subsubsection{Trial repetitions}

The effect of repetitions, or trial number, is summarised in Table 3 in which the entry in the $i^{\text {th }}$ row and $j^{\text {th }}$ column represents the $S_{P C A}$ value between the $i^{\text {th }}$ and $j^{\text {th }}$ trials. The diagonal terms are necessarily 100, and as all of the $S_{P C A}$ values represent most of the energy in the waveform (i.e. values $>98$ ), the changes in paths traced by the subjects across trials were small. The largest differences, as expected, were between the first and last trials, the paths of which are presented in Figure 6.

\subsection{Variability in reaching path}

The preliminary $S_{P C A}$ analysis identified three factors affecting the reaching motion patterns: age group, direction and type. PCA analysis was used to extract the principal components of reaching paths within the eight sets formed by taking the combinations of the two groups in each of these three categories. For all eight sets, three principal components were sufficient to capture more than $99 \%$ of the covariance in the observed data, suggesting low vari-
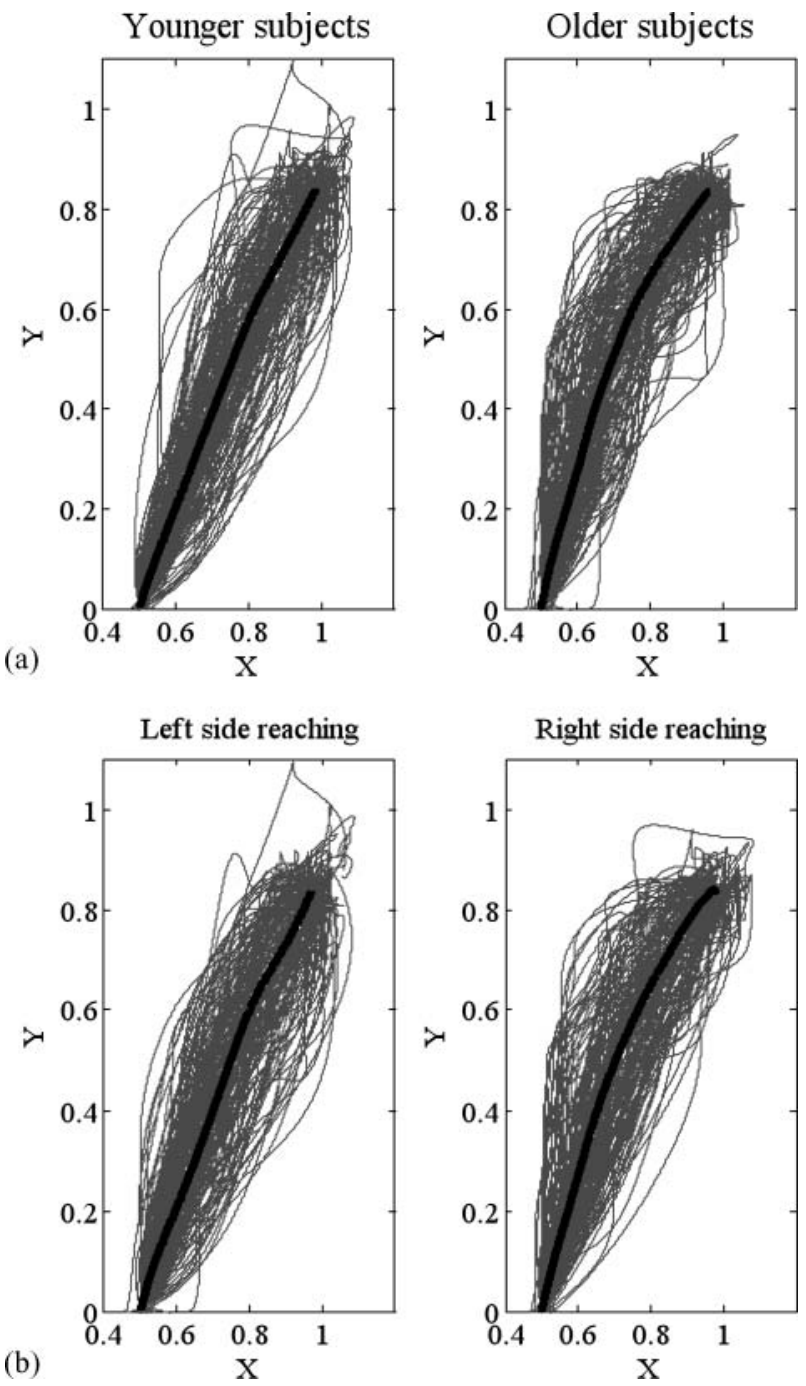

Figure 4. Paths traced for groups listed in Table 1 shown in grey with average paths shown in black. (a) Paths traced by the older (right) subjects were in general more curved than those traced by the younger (left) subjects. (b) Paths for reaching toward the left (left) and right (right) sides were different.

ability in the intraset data, as expected. Average paths for the eight sets were computed according to Equation (2). The signed area between each path traced by a subject and the average path corresponding to the appropriate set was used as the measure of variability. Statistical analysis was carried out with the signed area as the response variable and age group, direction, dominance and type as factors. Subjects were included as a random factor to account for inter-subject differences in the variability. Trial number was included as a covariate in the analysis. The multivariate regression model obtained from the statistical analysis had a Pearson correlation coefficient of 0.51 between the data and the fitted value.

Large inter-subject differences were observed ( $p=$ $.000)$ in the variability. The variability was also affected 

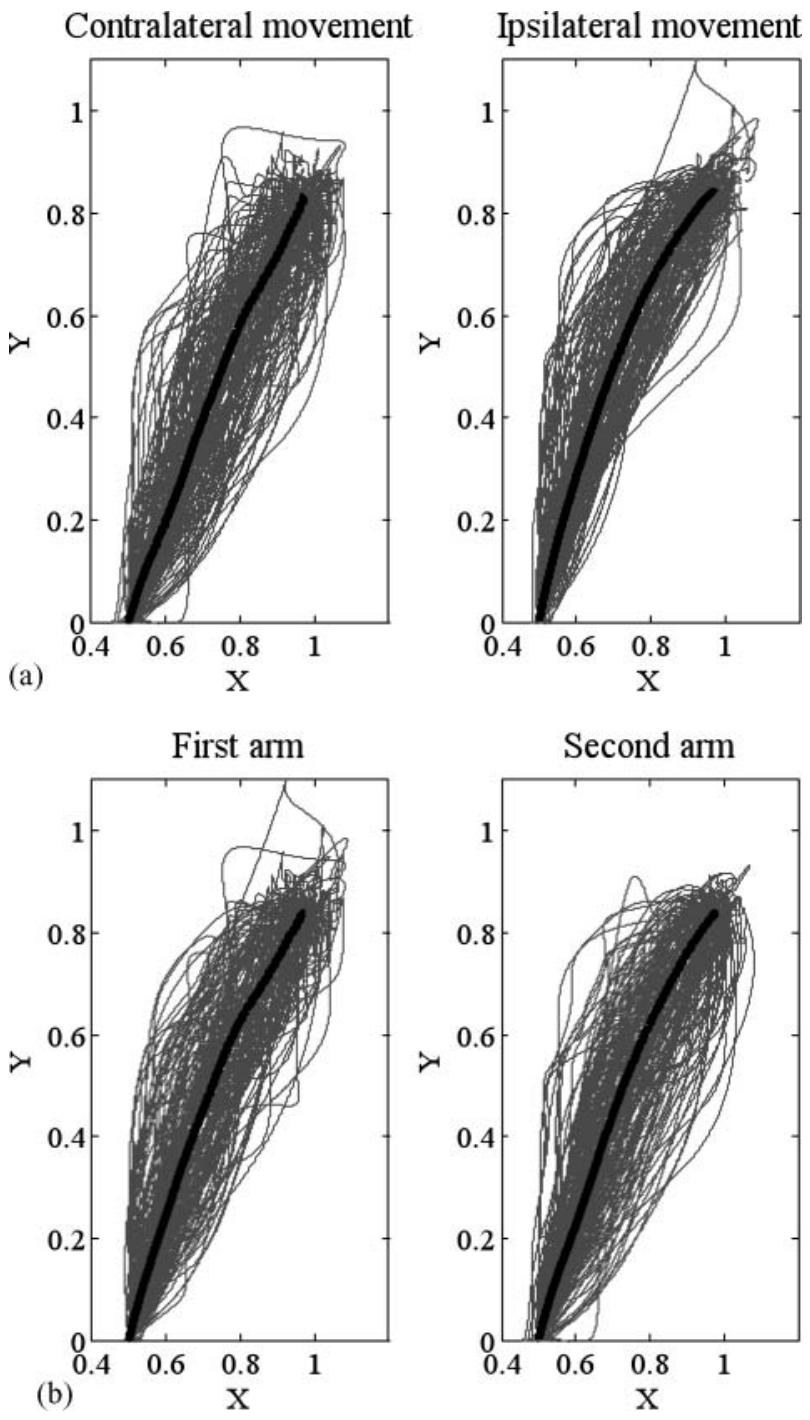

Figure 5. Paths traced for groups listed in Table 1 shown in grey with average paths shown in black. (a) The paths in ipsilateral (right) reaching were in general more curved than the paths in contralateral reaching (left). (b) First (left) and second (right) arm paths were in general quite similar.

by the trial number $(p=.009)$ and was different for the first and second arm $(p=.043)$. Other factors, including age group $(p=992)$, direction $(p=.979)$, dominance $(p=.779)$ and type $(p=.980)$ had no significant effect. Therefore, the important factors that affected variability in reaching path for this cohort were the trial number and whether the arm was first or second to perform motion. The coefficient of the term corresponding to trial number was negative, suggesting that the variability decreased as more motions were performed. This trend can also be seen in Figure 6, where the paths for the first reaching motion are more scattered than the paths for the fifth reaching motion. Bonferroni post hoc analysis revealed that the arm with which movement was performed first had higher variability, indicating a learning effect.
Table 3. $S_{P C A}$ between the paths for different trial numbers; $(i, j)$ entry lists the similarity between the $i$ th and $j$ th trial

\begin{tabular}{lccccc}
\hline Trial no. & 1 & 2 & 3 & 4 & 5 \\
\hline 1 & 100 & 99.3 & 98.7 & 99.1 & 98.2 \\
2 & 99.3 & 100 & 99.4 & 99.4 & 99.4 \\
3 & 98.7 & 99.4 & 100 & 99.2 & 99.5 \\
4 & 99.1 & 99.4 & 99.2 & 100 & 98.8 \\
5 & 98.2 & 99.4 & 99.5 & 98.8 & 100 \\
\hline
\end{tabular}

\subsection{Velocity profile smoothness}

To determine the important factors that affect the smoothness of the velocity profile, statistical analysis was carried out with $N^{*}$ as the response variable. Age group, direction, dominance and type were included as factors, and subjects were included as a random factor to account for inter-subject differences. Trial number was included as a covariate to investigate if performing more motions affected smoothness. This statistical analysis indicated that the significant factors $(p<.05)$ affecting movement smoothness were age group ( $p=.006)$, type $(p=.022)$ and trial number $(p=.000)$. All other factors, direction $(p=.872)$, dominance $(p=.796)$ and order $(p=.927)$, were not statistically significant. The Pearson correlation coefficient between the observed data and fitted value was 0.724 , indicating a good fit. Bonferroni post hoc analysis showed that the contralateral movements were smoother than the ipsilateral movements.

The smoothness of the velocity profile can be associated with the subjects learning to perform the reaching motions with the X-Y table. Figure 7 shows that the arm motions became smoother as more motions were performed. The mean and standard deviations of smoothness in trials one
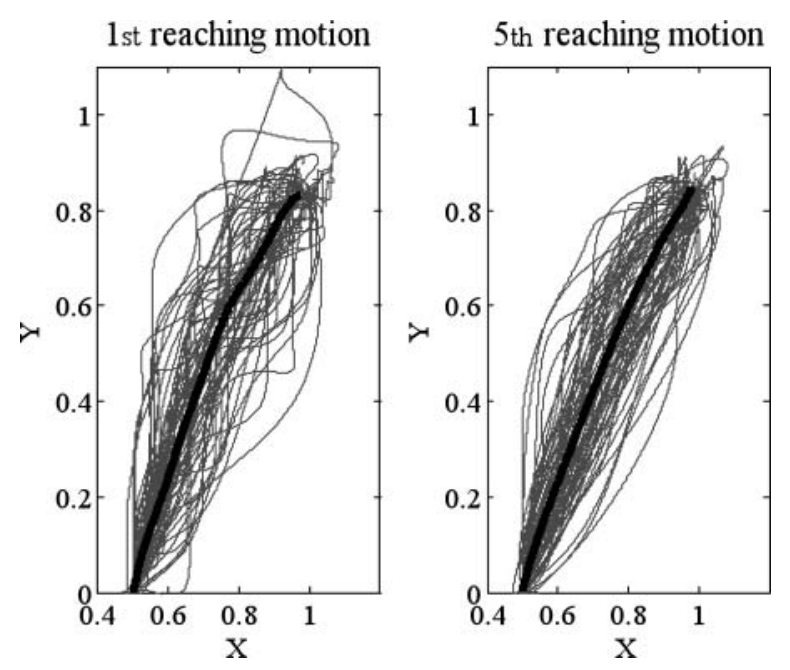

Figure 6. Paths traced for the 1st and 5th reaching trial shown in grey with the average paths shown in black. The reaching paths for the first and the last trial numbers were similar. 


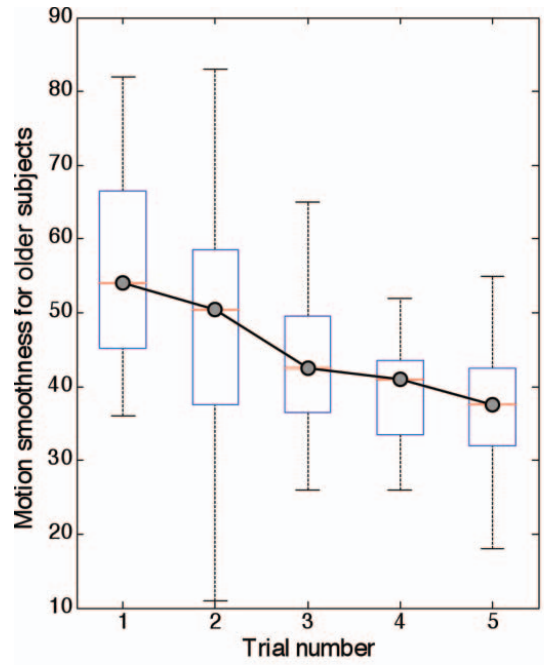

(a)

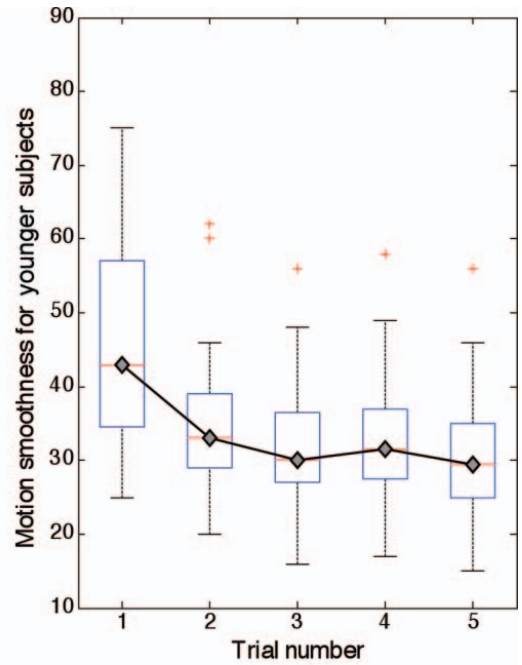

(b)

Figure 7. Motion smoothness computed as the number of modes required to capture $98 \%$ of the energy in velocity profile of reaching trials for the older (a) and the younger (b) subjects shown as a function of trial number.

through five for younger and older subjects are presented in Table 4. A two sample $t$-test was applied to compare the smoothness values of younger and older subjects in each trial. The $p$ values obtained from the $t$-test (listed in Table 4) show that the observed differences were statistically significant for all trials.

The younger group started with smoother velocity profiles than the older group and exhibited a rapid smoothing from trial one to trial two. By trial three, the younger group reached a roughly constant value of the smoothness metric. In contrast, the older group exhibited a slower improvement in smoothness, with adaptation seemingly still underway at the fifth trial. Therefore, the younger subjects performed smoother movements overall when compared with the older group and adapted more quickly to the motion and/or use of the X-Y table.

\section{Discussion}

As evident, the data analysis methods developed and evaluated with the X-Y table are able to capture differences between the arm reaching patterns of younger and older

Table 4. Velocity profile smoothness (mean, standard deviation) for younger and older subjects during trials one to five. $p$ value obtained from $t$-test for comparison of younger and older subjects.

\begin{tabular}{lllr}
\hline Trial no./group & Younger & Older & $p$ value \\
\hline 1 & $(46.09,14.51)$ & $(56.26,12.83)$ & 0.005 \\
2 & $(34.72,9.51)$ & $(49.97,15.19)$ & 0.000 \\
3 & $(31.22,8.19)$ & $(43.00,9.37)$ & 0.000 \\
4 & $(32.63,9.36)$ & $(39.38,7.09)$ & 0.002 \\
5 & $(31.00,8.69)$ & $(38.00,8.85)$ & 0.002 \\
\hline
\end{tabular}

subjects. The $S_{P C A}$ metric used to capture similarity in the path data from any two different groups, such as younger and older subjects, overcomes some of the limitations of other current metrics that rely on assumed straight-line paths. The most significant drawback of existing methods is that they do not quantify similarity, so two paths exhibiting equal values of existing metrics are not necessarily similar. As the $S_{P C A}$ value compares the reaching paths in two groups by computing similarity between the principal components, two dissimilar data sets cannot have $S_{P C A}$ values near 100. As a data-driven quantitative metric, $S_{P C A}$ is a more objective measure than the commonly used ordinal and qualitative assessment tools. This is, however, also the most significant limitation of $S_{P C A}$. As a quantitative metric, it requires rich data sets of arm reaching motions. Furthermore, $S_{P C A}$ is heuristic in nature, and the parameter selections (e.g. \% of the covariance considered) depend on the type of data set and application. Similarly, the Fourierbased smoothness metric has the advantage of requiring only one differentiation of position data and better distinguishing the differences in smoothness between two unlike paths than other existing metrics. As with $S_{P C A}$, though, this smoothness metric requires intuitive judgements to specify the percentage of energy content in the data to be captured with the Fourier series. In both cases, selection of a large percentage is an adequate heuristic.

The $S_{P C A}$ analysis revealed that the shape of the paths traced by the younger subjects differed from those traced by the older subjects, with the older subjects in general tracing more curved paths. Interestingly, this result is in contrast to the findings of Ketcham et al. (2002) and Lee et al. (2007), in which no significant differences in path shape were observed between older and younger subjects for line tracing. The present finding is, however, consistent with the results 
of Cesqui et al. (2008) in which age-related differences were observed in cyclic line tracing performed in the presence of a novel force field while holding the manipulandum of a robotic device. It may be that the additional muscle forces required because of the interaction with the robotic devices in these experiments are the source of the difference in path shape since no such difference is observed in line tracing without a robot. This explanation is consistent with the observations of Lee et al. (2007) that older subjects sacrificed smoothness of their velocity profiles in tracing tasks in order to reduce the total torque requirements. Similarly, Cesqui et al. (2008) found that older subjects pursued a different adaptation strategy than younger subjects, which involved increased shoulder usage and different modulation of joint torques.

In terms of variability in reaching motion, the signed area between the individual paths and the average paths formed by grouping the data according to like combinations of age group, movement type and reaching direction did not indicate any significant difference between the younger and older subjects. Rather, the only statistically significant differences were observed between motions of the first and second arm and between motions performed early and late in the study, with the second arm and later motions exhibiting lower variability. Therefore, the results suggest no degradation of repeatability in reaching tasks with age. They do, however, point to the importance of adaptation to the task over the course of the subject's participation. With lower variability observed in the second arm, some transfer of adaptation appears to have taken place from the first to the second arm. Additionally, the improvement in the smoothness metric with trial number for both younger and older subjects indicates adaptation with repetition.

Independent of trial number, the younger subjects traced smoother paths than the older subjects. The younger subjects both exhibited smoother motions in the initial trials and more rapidly converged to a relatively stable value of the smoothness metric over the course of the experiment. The older subjects executed smoother paths as the experiment progressed but never reached the smoothness level of the younger subjects. Cesqui et al. (2008) showed a similar degradation of smoothness in the multi-joint reaching movements of older subjects. The superiority of the younger subjects in terms of motion smoothness and adaptation may be due to the same issue identified above of older subjects minimising joint torque. If older subjects are optimising for this alternative objective, the results would not necessarily indicate degradation with age in the ability to trace smooth paths and rapidly adapt. Muscle strength may simply be the more dominant factor.

This paper proposes that the data acquisition and analysis methods described herein can be used for diagnosis and assessment of neurorehabilitation patients. As the results of this paper show differences in both reaching path and smoothness between younger and older subjects, when assessing stroke-related effects, it is important to evaluate the motion patterns of stroke patients relative to those of age-matched controls. $S_{P C A}$ quantifies similarity in path shape, and the Fourier-based metric quantifies smoothness. Larger values of $S_{P C A}$ and fewer modes of a Fourier series required to capture $98 \%$ of the path energy are expected to characterise a more nearly normal reaching pattern. Future studies will validate the use of these methods developed with the X-Y table in evaluation of recovery from stroke during the acute phase of rehabilitation.

\section{References}

Beppu H, Suda M, Tanaka R. 1984. Analysis of cerebellar motor disorders by visually guided elbow tracking movement. Brain. 107:787-809.

Brooks VB, Cooke JD, Thomas JS. 1973. The continuity of movements. In: Stein RB, editor. Control of posture and locomotion. New York (NY): Plenum:. p. 257-272.

Buch ER, Young S, Contreras-Vidal JL. 2003. Visuomotor adaptation in normal aging. Learn Mem. 10(1):55-63.

Burgar CG, Lum PS, Shor PC, Van der Loos M. 2000. Development of robots for rehabilitation therapy: The Palo Alto VA/Stanford experience. J Rehabil Res Dev. 37:663-673.

Celik O, O’Malley MK, Boake C, Levin HS, Yozbitiran N, Reistetter T. (2010). Normalized movement quality measures for therapeutic robots strongly correlate with clinical motor impairment measures. IEEE Trans Neural Sys Rehab Eng. 18(4):433-444.

Cesqui B, Macrì G, Dario P, Micera S. 2008. Characterization of age-related modifications of upper limb motor control strategies in a new dynamic environment. J Neuroeng Rehabil. 5:31-44.

Colombo R, Pisano F, Mazzone A, Delconte C, Micera S, Carrozza MC, Dario P, Minuco G. 2007. Design strategies to improve patient motivation during robot-aided rehabilitation. J Neuroeng Rehabil. 4:3-15.

Colombo R, Pisano F, Micera S, Mazzone A, Delconte C, Carrozza MC, Dario P, Minuco G. 2005. Robotic techniques for upper limb evaluation and rehabilitation of stroke patients. IEEE Trans Neural Sys Rehab Eng. 13(3):311-324.

Crear DE. 2006. Design of an X-Y table for investigating and rehabilitating human motor control [Undergraduate thesis]. The Ohio State University.

Flash T, Hogan N. 1985. The coordination of arm movements: an experimentally confirmed mathematical model. J Neurosci. 5:1688-1703.

Forner-Cordero A, Levin O, Li Y, Swinnen SP. 2005. Principal component analysis of complex multi-joint coordinative movements. Biol Cybern. 93(1):63-78.

Heuer H, Hegele M. 2008. Adaptation to direction-dependent visuo-motor rotations and its decay in younger and older adults. Acta Psychologica. 127:369-381.

Keith RA, Granger CV, Hamilton BB, Sherwin FS. 1987. The functional independence measure: a new tool for rehabilitation. Adv Clinic Rehab. 1:6-18.

Ketcham CJ, Dounskaia N, Stelmach GE. 2003. Control of multijoint drawing movements: a comparison of young and elderly adults. Proceedings of the 1st International IEEE EMBS Conference on Neural Engineering; Capri Island, Italy. p. 249252 . 
Ketcham CJ, Seidler RD, Van Gemmert AWA, Stelmach GE. 2002. Age-related kinematic differences as influenced by task difficulty, target size, and movement amplitude. J Geron Psy Sci. 57b(1):54-64.

Kitagawa G, Gersch W. 1985. A smoothness priors long AR model method for spectral estimation. IEEE Trans Auto Ctrl. 30(1): $57-65$.

Krzanowski WJ. 1979. Between-groups comparison of principal components. J Am Stat Assoc. 74(367):703707.

Lee G, Fradet L, Ketcham CJ, Dounskaia N. 2007. Efficient control of arm movements in advanced age. Exp Brain Res. 177:7894.

Lum PS, Burgar CG, Shor PC, Majmundar M, Van der Loos M. 2002. Robot-assisted movement training compared with conventional therapy techniques for the rehabilitation of upperlimb motor function after stroke. Arch Phys Med Rehabil. 83:952-959.

Lum PS, Mulroy S, Amdur RL, Requejo P, Prilutsky BI, Dromerick AW. 2009. Gains in upper extremity function after stroke via recovery or compensation: Potential differential effects on amount of real-world limb use. Top Stroke Rehabil. 16(4):237-253.

Morris DM, Uswatte G, Crago JE, Cook EW, Taub E. 2001. The reliability of the Wolf Motor Function Test for assessing upper extremity function after stroke. Arch Phys Med Rehabil. 82(6):750-755.
Muniz AMS, Nadal J. 2007. Application of principal component analysis in vertical ground reaction force to discriminate normal and abnormal gait. Gait Pos. 29:31-35.

Nicoletti G, Arabia G, Pugliese P, Torchia G, Pucci F, Gambardella A, Quattrone A, Zappia M. 2005. Movement time and aging: a normative study in healthy subjects with the "Movement Time Analyzer". Aging Clin Exp Res. 17:207-210.

Olivier I, Audiffren M, Ripoll H. 1998. Age-related differences in the preparatory processes of motor programming. J Exp Child Psychol. 69(1):49-65.

Rohrer B, Fasoli S, Krebs HI, Hughes R, Volpe B, Frontera WR, Stein J, Hogan N. 2002. Movement smoothness changes during stroke recovery. J Neurosci. 22(18):8297-8304.

Smith CD, Umberger GH, Manning EL, Slevin JT, Wekstein DR, Schmitt FA, Markesbery WR, Zhang Z, Gerhardt GGA, Kryscio RJ, et al. 1999. Critical decline in fine motor hand movements in human aging. Neurology. 53:1458-1461.

Teulings HL, Contreras Vidal JL, Stelmach GE, Alder CH. 1997. Parkinsonism reduces coordination of fingers, wrist, and arm in fine motor control. Exp Neurol. 146:159-170.

Volpe B, Krebs H, Hogan N, Edelstein L, Diels C, Aisen M. 2000. A novel approach to stroke rehabilitation: robot-aided sensorimotor stimulation. Neurology. 54:1938-1944.

Yang K, Shahabi C. 2004. A PCA-based similarity measure for multivariate time series. Proceedings of the 2nd ACM International Workshop on Multimedia Databases; Washington, DC, USA. p. $65-74$. 

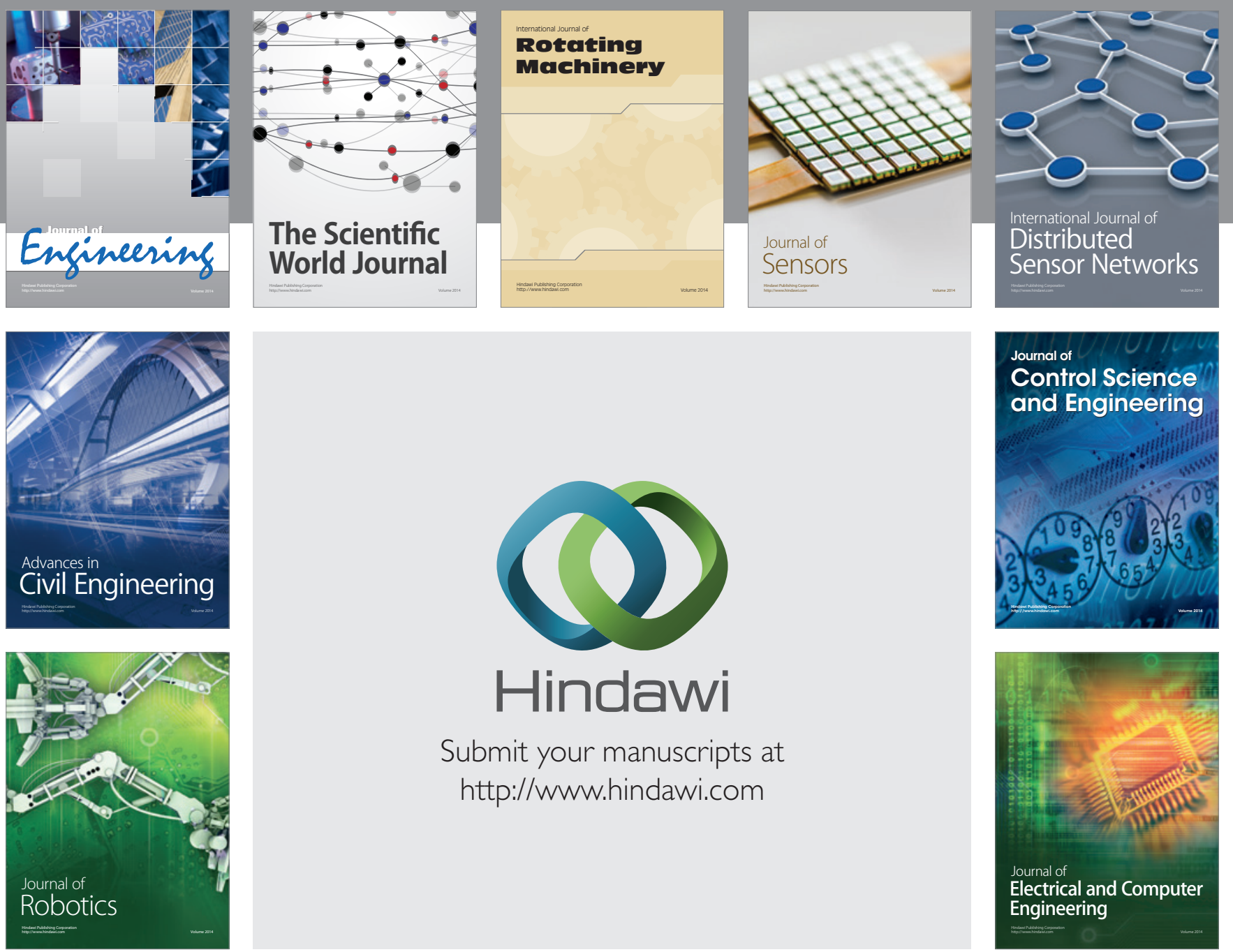

Submit your manuscripts at

http://www.hindawi.com
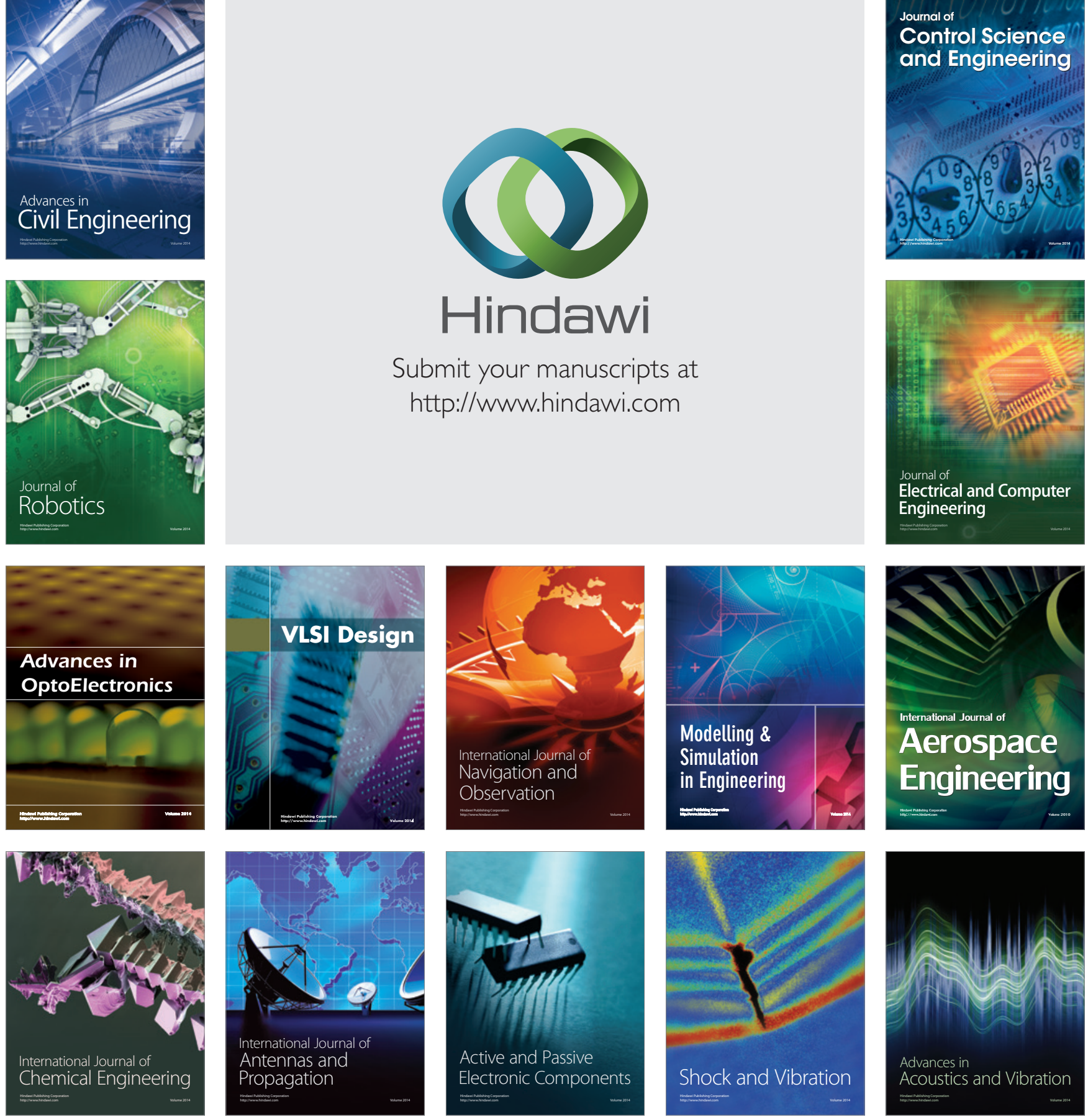